\section{Sichere Alternative zur Lobektomie}

\section{Eine intraoperative Brachytherapie könnte die Rezidivraten nach einer sublobaren Resektion bei Patienten mit nichtkleinzelligem Lungenkarzinom (NSCLC) reduzieren. Wie hoch ist das Risiko für Nebenwirkungen?}

B ei Patienten mit NSCLC im frühen Stadium und reduzierter Lungenfunktion sowie erhöhtem Risiko ist die sublobare Resektion eine Alternative zur Lobektomie, allerdings mit höheren Rezidivraten. Wie effektiv und sicher eine zusätzliche intraoperative Brachytherapie ist, wurde in der multizentrischen PhaseIII-Studie Z4032 der American College of Surgeons Oncology Group (ACOSOG) untersucht: es wurde die sublobare Resektion mit oder ohne intraoperative Brachytherapie bei Hochrisiko-Patienten mit NSCLC Stadium I verglichen. Die Daten von 222 Patienten zur Sicherheit liegen nun vor. Sie sind insofern interessant, weil nicht operative Therapien wie die stereotaktische Bestrahlung und die Radiofre-

\section{K-Ras-Status variabel}

In einer Metaanalyse wurde untersucht, ob sich bei primärem und metastasiertem nicht kleinzelligem Lungenkarzinom (NSCLC) EGFR- und K-Ras-Genotypen unterscheiden.

$\mathrm{D}$ er epidermale Wachstumsfaktorrezeptor (EGFR) wird in 60-90\% der NSCLC-Tumoren exprimiert. Bestimmte Mutationen sind mit besserer Prognose und dem Ansprechen auf EGFR-Tyrosinkinasehemmer (TKI) assoziiert. Außerdem könnten sich Expression und Amplifikation von EGFR zur Vorhersage der Wirksamkeit von TKIs eignen. Allerdings profitieren auch Patienten mit fortgeschrittenem NSCLC ohne EGFR-Expression und ein Teil der Patienten mit Wildtyp-EGFR davon. Eine mögliche Erklärung wären Unterschiede zwischen Primärtumor und Metastasen, da im Rahmen der Metastasierung Genveränderungen und Mutationen auftreten können.

Mutationen im K-Ras-Gen korrelieren mit schlechten Überlebensraten. Außerdem weisen sie auf die Unwirksamkeit der Therapie mit einem EGFR-TKI hin. quenzablation zunehmend Beachtung finden, selbst bei operablen Patienten.

30- und 90-Tage-Mortalität waren in beiden Gruppen vergleichbar. Drei Patienten $(1,4 \%)$ starben innerhalb von 30 Tagen, drei weitere innerhalb der folgenden 60 Tage. Vier dieser sechs Todesfälle gingen auf die Operation zurück.

Nebenwirkungen der Grade 3/4 waren in beiden Gruppen ähnlich häufig ( $\mathrm{Grad}$ 3: 25,4 bzw. 30,6\%; Grad 4: 7 bzw. 7,4\%), am häufigsten Hämorrhagien, Dyspnoe und Hypoxie. Zum Vergleich: In der kürzlich durchgeführten Phase-II-Studie der Radiation Therapy and Oncology Group zur stereotaktischen Bestrahlung waren die Nebenwirkungsraten niedriger, allerdings sind die Populationen nicht direkt

In einer Metaanalyse von 14 Studien wurden Mutationen, Amplifikation und Expression von EGFR und K-Ras-Mutationen bei primärem und metastasiertem NSCLC untersucht. Die Häufigkeit von EGFR-Mutationen war bei Primärtumoren und Metastasen vergleichbar (17,2 bzw. 19,9\%; $\mathrm{p}=0,31)$. In metastasierten Tumoren lagen tendenziell mehr EGFRKopien vor. Die Proteinexpression war tendenziell in primären Tumoren höher.

Die Häufigkeit von $K$-Ras-Mutationen war in Primärtumoren tendenziell höher (25,9 bzw. 18,7\%; p = 0,09), d. h. K-RasMutationen könnten während der Metastasierung verloren gehen. Eine weitere Möglichkeit wäre, dass Tumorzellen mit $K$-Ras-Wildtyp eher zur Metastasierung neigen als Tumoren mit mutiertem $K$-Ras.

Der K-Ras-Mutationsstatus im Gewebe eines primären NSCLC könnte also ein besserer Biomarker für die Auswahl von Patienten für eine Therapie mit EGFRTKIs sein als der in Metastasen.

Fazit: Bei primärem NSCLC scheint die kombinierte Detektion von EGFR- und $K$-Ras-Mutationen optimal zu sein, um Patienten für die Erstlinientherapie mit einem TKI auszuwählen. Judith Neumaier vergleichbar, und ein Vergleich der Wirksamkeit beider Methoden fehlt.

Bei einer Kohlenmonoxid-Diffusion der Lunge (DCLO) < 46\% war die Inzidenz von Grad-3-Nebenwirkungen signifikant höher (nach 30 Tagen Odds Ratio [OR] $0,97, \mathrm{p}=0,003$; nach 90 Tagen OR 0,98, p $=0,05)$. Außerdem ging die segmentale Resektion mit mehr Grad-3-Nebenwirkungen einher als die Wedge-Resektion.

Fazit: Die intraoperative Brachytherapie zusätzlich zur sublobaren Resektion wirkte sich nicht negativ auf die Morbidität aus und ist bei NSCLC-Patienten mit hohem Risiko sicher mit niedriger 30und 90-Tage-Mortalität. Judith Neumaier

Fernando $\mathrm{HC}$ et al. Thirty- and ninety-day outcomes after sublobar resection with and without brachytherapy for non-small cell lung cancer: results from a multicenter phase III study. J Thorac Cardiovasc Surg. 2011; 142(5):1143-51.

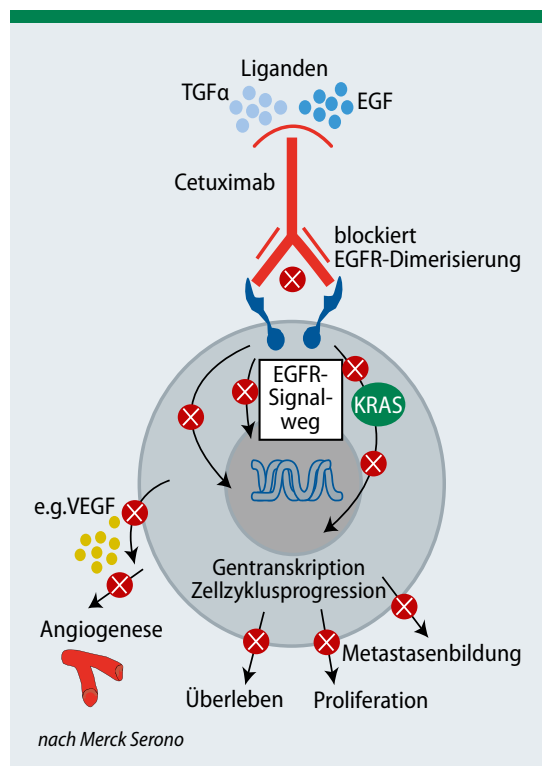

Ist das K-Ras-Protein mutiert, treibt es trotz Blockade des EGFR-Rezeptors das Tumorwachstum an. Eine EGFR-Therapie ist deshalb nur bei $K$-Ras-Wildtyp und bestimmten EGFR-Mutationen sinnvoll.

Han C et al. EGFR mutations, gene amplification, and protein expression and KRAS mutations in primary and metastatic tumors of nonsmall cell lung cancers and their clinical implications: a meta-analysis. Cancer Invest. 2011; 29(9):626-34. 